MATHEMATICS OF COMPUTATION

Volume 68, Number 227 , Pages 1013-1024

S 0025-5718(99)01054-6

Article electronically published on February 19, 1999

\title{
FINITE ELEMENT APPROXIMATION TO A CONTACT PROBLEM IN LINEAR THERMOELASTICITY
}

\author{
M. I. M. COPETTI
}

\begin{abstract}
A finite element approximation to the solution of a one-dimensional linear thermoelastic problem with unilateral contact of the Signorini type and heat flux is proposed. An error bound is derived and some numerical experiments are performed.
\end{abstract}

\section{INTRODUCTION}

We shall consider the numerical approximation of the following one-dimensional evolution problem with unilateral contact of the Signorini type:

$$
\begin{array}{ll}
D^{2} \tilde{\theta}=\tilde{\theta}_{t}+a(D u)_{t} & x \in I, t>0, \\
D^{2} u=a D \tilde{\theta} & x \in I, t>0, \\
\tilde{\theta}(x, 0)=p(x) & x \in I, \\
\tilde{\theta}(0, t)=\theta_{A} & t>0, \\
-D \tilde{\theta}(1, t)=k \tilde{\theta}(1, t) & t>0, \\
u(0, t)=0 & t>0, \\
u(1, t) \leq g, \quad D u(1, t) \leq a \tilde{\theta}(1, t) & t>0, \\
(D u(1, t)-a \tilde{\theta}(1, t))(u(1, t)-g)=0 & t>0,
\end{array}
$$

where $\tilde{\theta}(x, t)$ and $u(x, t)$ are the temperature and the displacement (parallel to the x-axis) of a homogeneous elastic body $A B$. The interval $I=(0,1)$ is the reference configuration of the body at the reference temperature $\tilde{\Theta}_{r}=0$. The coupling constant $a$ is usually small and is given in terms of physical parameters.

Here, $D \stackrel{\text { def }}{=} \frac{\partial}{\partial x}$, and $g>0$ is a constant representing the gap between the end $B(x=1)$ and an obstacle at temperature $\tilde{\theta}=0$. At the end $A(x=0)$, the body has a constant temperature $\theta_{A}$ and it is clamped. The right end $x=1$ is free to expand or contract and the body may be in contact with the obstacle or not. However, the position of the right edge at $t>0$ is not known a priori and the displacement cannot be more than $g$. A heat flux between the body and the obstacle is allowed with a constant heat transfer coefficient $k \geq 0$. The process is

Received by the editor May 7, 1997 and, in revised form, January 6, 1998 .

1991 Mathematics Subject Classification. Primary 65N30, 65N15.

Key words and phrases. Thermoelasticity, finite element method.

This work was partially supported by CNPq (grant 300766/92).

(C)1999 American Mathematical Society 
assumed to be slow and the acceleration term, present in the equations of linear thermoelasticity, has been omitted in (1.1b) leading to a quasi-static problem ([2]). We refer to Carlson ([3]) and Day ([6]) for physical background and mathematical modelling. Existence results to this problem were obtained by Andrews et al. in [1] using a heat transfer coefficient which may depend on the contact pressure and the distance of the right edge from the obstacle. Some numerical experiments using a finite difference scheme were performed by Shi et al. in [11].

Existence, uniqueness and regularity results to the solution of the contact problem with the temperature of the body being constant at both ends have been established by Copetti and Elliott in [4], Gilbert et al. in [9] and Shi and Shillor in [10]. In [4] Copetti and Elliott have also proposed and analysed a finite element approximation. The quasi-static problem with the body clamped at the boundary was studied by Day in [6]. The static case with unilateral Signorini boundary condition together with heat flux was investigated by Duvaut in [7] and an existence result to the full dynamic problem was obtained by Elliott and Qi in [8].

In this paper, we shall follow the work of Copetti and Elliott ([4]).

Following the idea introduced by Shi and Shillor in [10] we shall reformulate the problem into a decoupled form. Let us assume that the problem (1.1a)-(1.1h) has a classical solution. Integrating (1.1b) from $x$ to 1 we obtain

$$
D u(1, t)-D u(x, t)=a(\tilde{\theta}(1, t)-\tilde{\theta}(x, t)), \quad 0 \leq x \leq 1, t>0,
$$

and another integration with respect to $x$, from 0 to $x$, together with (1.1f) yields

$$
x D u(1, t)-u(x, t)=a \int_{0}^{x}(\tilde{\theta}(1, t)-\tilde{\theta}(\xi, t)), d \xi \quad 0 \leq x \leq 1, t>0,
$$

and, in particular,

$$
u(1, t)=D u(1, t)-a \int_{0}^{1}(\tilde{\theta}(1, t)-\tilde{\theta}(x, t)) d x .
$$

From (1.1h) we have

$$
\left(u(1, t)-a \int_{0}^{1} \tilde{\theta}(x, t) d x\right)(u(1, t)-g)=0
$$

and the condition $D u(1, t) \leq a \tilde{\theta}(1, t)$ implies

$$
u(1, t) \leq a \int_{0}^{1} \tilde{\theta}(x, t) d x .
$$

Therefore

$$
u(1, t)=\min \left\{a \int_{0}^{1} \tilde{\theta}(x, t) d x, g\right\}
$$

and

$$
\begin{aligned}
D u(1, t) & =\min \left\{a \int_{0}^{1} \tilde{\theta}(x, t) d x, g\right\}+a \int_{0}^{1}(\tilde{\theta}(1, t)-\tilde{\theta}(x, t)) d x \\
& =-\max \left\{a \int_{0}^{1} \tilde{\theta}(x, t) d x-g, 0\right\}+a \tilde{\theta}(1, t) .
\end{aligned}
$$


Using this result in equation (1.2) gives

$$
D u(x, t)=-\max \left\{a \int_{0}^{1} \tilde{\theta}(x, t) d x-g, 0\right\}+a \tilde{\theta}(x, t) .
$$

Differentiating this equation with respect to $t$ we obtain

$$
(D u)_{t}(x, t)=a \tilde{\theta}_{t}(x, t)-\frac{d}{d t} \max \left\{a \int_{0}^{1} \tilde{\theta}(x, t) d x-g, 0\right\}
$$

and equation (1.1a) may be written as

$$
\left(1+a^{2}\right) \tilde{\theta}_{t}=D^{2} \tilde{\theta}+a^{2} \frac{d}{d t} \max \left\{\int_{0}^{1} \tilde{\theta}(x, t) d x-\frac{g}{a}, 0\right\} .
$$

Recalling (1.3) and (1.5) we find that the displacement is given by

$$
u(x, t)=-a x \max \left\{\int_{0}^{1} \tilde{\theta}(x, t) d x-\frac{g}{a}, 0\right\}+a \int_{0}^{x} \tilde{\theta}(\xi, t) d \xi .
$$

The following existence and uniqueness result was obtained by Andrews et al. in $[1]$ :

Theorem 1.1. Given $p(x) \in H^{1}(I)$ with $p(0)=\theta_{A}$ and $k \in C^{1}(\mathbf{R}), k \geq 0$, there exists a unique $\tilde{\theta} \in H^{2,1}\left(\Omega_{T}\right)$ satisfying

$$
\begin{array}{ll}
\left(1+a^{2}\right) \tilde{\theta}_{t}=D^{2} \tilde{\theta}+a^{2} \frac{d}{d t} \max \left\{\int_{0}^{1} \tilde{\theta}(x, t) d x-\frac{g}{a}, 0\right\}, & \text { a.e. in } \Omega_{T}, \\
\tilde{\theta}(x, 0)=p(x), & x \in I, \\
\tilde{\theta}(0, t)=\theta_{A}, & 0<t<T, \\
-D \tilde{\theta}(1, t)=k \tilde{\theta}(1, t), & \text { a.e. in }(0, T),
\end{array}
$$

where $\Omega_{T}=I \times(0, T)$, provided $0<a<1$.

Remark 1.2. In the work of Andrews et al., in [1], $\theta_{A}=1$ and the heat transfer coefficient $k(\cdot)$ is a function of $g-u(1, t)+\tilde{\sigma}(t)$, where $\tilde{\sigma}(t)=D u(1, t)-a \tilde{\theta}(1, t)$.

Remark 1.3. It would be natural to consider the general case where the temperature at $x=0$ is time dependent and may be different from the value of the initial temperature at $x=0$. This was done by Copetti and Elliott in [4] when the temperature of the body is constant at both ends.

Letting $\theta(x, t)=\tilde{\theta}(x, t)+\theta_{A}(x-1)$ we obtain the contact problem with homogeneous boundary condition at $x=0$ for the temperature

$$
\begin{array}{ll}
\left(1+a^{2}\right) \theta_{t}-D^{2} \theta=a^{2} \frac{d}{d t}[\gamma]_{+}, & x \in I, t>0, \\
\gamma(t):=(1, \theta(t))-r, & t>0, \\
\theta(x, 0)=\theta_{0}(x)=p(x)+\theta_{A}(x-1), & x \in I, \\
-D \theta(1, t)=k \theta(1, t)-\theta_{A}, & t>0, \\
\theta(0, t)=0, & t>0,
\end{array}
$$

where $(\cdot, \cdot)$ is the $L^{2}$-inner product, $[\gamma(t)]_{+}:=\max \{\gamma(t), 0\}$ and $r=\frac{g}{a}-\frac{\theta_{A}}{2}$. Note that $\tilde{\sigma}(t)=-a[\gamma(t)]_{+}$.

Throughout this paper, we denote the norms of $L^{2}(I)$ and $H^{s}(I)$ by $\|\cdot\|$ and $\|\cdot\|_{s}$, respectively. The semi-norm $\|D v\|$ is indicated by $|v|_{1}$. 


\section{The finite element approximation of $\{\theta, \gamma\}$}

Integrating (1.7a) against test functions $\chi$ in $H_{E}^{1}(I)=\left\{v \in H^{1}(I) \mid v(0)=0\right\}$ and using the boundary conditions we obtain

$$
\left(1+a^{2}\right)\left(\theta_{t}, \chi\right)+(D \theta, D \chi)+k \theta(1, t) \chi(1)=\theta_{A} \chi(1)+a^{2} \frac{d}{d t}[\gamma]_{+}(1, \chi) .
$$

Let $0=x_{0}<x_{1}<\ldots<x_{s}=1$ be an equidistant partition of the interval $(0,1)$ into subintervals $I_{j}=\left(x_{j-1}, x_{j}\right), j=1, \ldots, s$, of length $h=\frac{1}{s}$ and denote by $S_{E}^{h}$ the finite element space

$$
S_{E}^{h}=\left\{\chi \in C^{0}(\bar{I}):\left.\chi\right|_{I_{j}} \in P_{1}, \chi(0)=0\right\},
$$

where $P_{1}$ is the space of linear functions.

The discrete Galerkin method for (1.7a)-(1.7e) is to find $\Theta^{n} \in S_{E}^{h}$ and $\Gamma^{n}$, $n=1, \ldots, N$, such that $\forall \chi \in S_{E}^{h}$

$$
\begin{gathered}
\left(1+a^{2}\right)\left(\frac{\Theta^{n}-\Theta^{n-1}}{\Delta t}, \chi\right)+\left(D \Theta^{n}, D \chi\right)+k \Theta^{n}(1) \chi(1) \\
=\theta_{A} \chi(1)+a^{2}\left(\frac{\left[\Gamma^{n}\right]_{+}-\left[\Gamma^{n-1}\right]_{+}}{\Delta t}, \chi\right), \\
\Gamma^{n}=\left(1, \Theta^{n}\right)-r,
\end{gathered}
$$

with $\Theta^{0}$ the $L^{2}$-projection of $\theta_{0}$ and $\Gamma^{0}=\gamma(0)$ and where $\Delta t=\frac{T}{N}$. This choice of $\Theta^{0}$ turns out to be convenient for the error analysis.

Given $\Theta^{n-1}$ we iterate to find $\Theta^{n}$ :

$$
\begin{aligned}
& \left(1+a^{2}\right)\left(\Theta_{l}^{n}, \chi\right)+\Delta t\left(D \Theta_{l}^{n}, D \chi\right)+\Delta t k \Theta_{l}^{n}(1) \chi(1) \\
& \quad=\left(1+a^{2}\right)\left(\Theta^{n-1}, \chi\right)+\Delta t \theta_{A} \chi(1)+a^{2}\left(\left[\Gamma_{l-1}^{n}\right]_{+}-\left[\Gamma^{n-1}\right]_{+}, \chi\right),
\end{aligned}
$$

where $\Gamma_{l-1}^{n}=\left(1, \Theta_{l-1}^{n}\right)-r$.

Theorem 2.1. There exists a unique sequence $\left\{\Theta^{n}\right\}_{n=1}^{N}$ solving (2.2a) and (2.2b).

Proof. Writing

$$
\Theta_{l}^{n}=\sum_{i=1}^{s} c_{l, i}^{n+1} \chi_{i}
$$

where $\left\{\chi_{i}\right\}_{i=1}^{s}$ is the piecewise linear basis for $S_{E}^{h}$, and taking $\chi=\chi_{j}, j=1, \ldots, s$, in (2.3) results in

$$
\begin{aligned}
(1+ & \left.a^{2}\right) \sum_{i=1}^{s} c_{l, i}^{n}\left(\chi_{i}, \chi_{j}\right)+\Delta t \sum_{i=1}^{s} c_{l, i}^{n}\left(D \chi_{i}, D \chi_{j}\right)+\Delta t k \sum_{i=1}^{s} c_{l, i}^{n} \chi_{i}(1) \chi_{j}(1) \\
= & \left(1+a^{2}\right) \sum_{i=1}^{s} c_{i}^{n-1}\left(\chi_{i}, \chi_{j}\right)+\Delta t \theta_{A} \chi_{j}(1)+a^{2}\left(\left[\Gamma_{l-1}^{n}\right]_{+}-\left[\Gamma^{n-1}\right]_{+}, \chi_{j}\right) .
\end{aligned}
$$

Hence, given $\Theta_{l-1}^{n}$, we have to solve the system

$$
\left(\left(1+a^{2}\right) M+\Delta t K+\Delta t k B\right) \underline{c}_{l}^{n}=\left(1+a^{2}\right) M \underline{c}^{n-1}+\Delta t \theta_{A} \underline{d}+a^{2} \underline{e},
$$


where

$$
\begin{gathered}
M_{i j}=\left(\chi_{i}, \chi_{j}\right), \quad K_{i j}=\left(D \chi_{i}, D \chi_{j}\right), \\
\underline{c}_{l}^{n}=\left\{c_{l, i}^{n}\right\}, \underline{c}^{n-1}=\left\{c_{i}^{n-1}\right\}, \\
B_{i j}=0 i, j \neq s, B_{s s}=1, \\
\{\underline{d}\}_{i}=0 i \neq s, \quad\{\underline{d}\}_{s}=1, \\
\{\underline{e}\}_{i}=\left(\left[\Gamma_{l-1}^{n}\right]_{+}-\left[\Gamma^{n-1}\right]_{+}\right) h i \neq s,\{\underline{e}\}_{s}=\left(\left[\Gamma_{l-1}^{n}\right]_{+}-\left[\Gamma^{n-1}\right]_{+}\right) \frac{h}{2} .
\end{gathered}
$$

Since $k \geq 0,\left(1+a^{2}\right) M+\Delta t K+\Delta t k B$ is invertible and the above system has a unique solution $\underline{c}_{l}^{n}$.

Defining $\mathcal{F}: \bar{S}_{E}^{h} \rightarrow S_{E}^{h}$ such that, for $\Theta \in S_{E}^{h}, \mathcal{F}(\Theta)$ satisfies, for any $\chi \in S_{E}^{h}$,

$$
\begin{aligned}
\left(1+a^{2}\right)(\mathcal{F}(\Theta), \chi)+\Delta t(D \mathcal{F}(\Theta), D \chi)+\Delta t k \mathcal{F}(\Theta)(1) \chi(1) & \\
= & \left(1+a^{2}\right)\left(\Theta^{n-1}, \chi\right)+\Delta t \theta_{A} \chi(1)+a^{2}\left([\Gamma(\Theta)]_{+}-\left[\Gamma^{n-1}\right]_{+}, \chi\right),
\end{aligned}
$$

where $\Gamma(\Theta)=(1, \Theta)-r$, it follows that $\mathcal{F}$ is well defined and (2.2a) and $(2.2 \mathrm{~b})$ has a unique solution if $\mathcal{F}$ has a unique fixed point.

Let us take $\Theta, \eta \in S_{E}^{h}$. By (2.4), $\forall \chi \in S_{E}^{h}$,

$$
\begin{gathered}
\left(1+a^{2}\right)(\mathcal{F}(\Theta)-\mathcal{F}(\eta), \chi)+\Delta t(D(\mathcal{F}(\Theta)-\mathcal{F}(\eta)), D \chi) \\
+\Delta t k(\mathcal{F}(\Theta)(1)-\mathcal{F}(\eta)(1)) \chi(1)=a^{2}\left([\Gamma(\Theta)]_{+}-[\Gamma(\eta)]_{+}, \chi\right) .
\end{gathered}
$$

Choosing $\chi=\mathcal{F}(\Theta)-\mathcal{F}(\eta)$, we obtain

$$
\begin{aligned}
\left(1+a^{2}\right) \| \mathcal{F}(\Theta)- & \mathcal{F}(\eta) \|^{2}+\Delta t|\mathcal{F}(\Theta)-\mathcal{F}(\eta)|_{1}^{2}+\Delta t k[\mathcal{F}(\Theta)(1)-\mathcal{F}(\eta)(1)]^{2} \\
& \leq a^{2}\left\|[\Gamma(\Theta)]_{+}-[\Gamma(\eta)]_{+}\right\|\|\mathcal{F}(\Theta)-\mathcal{F}(\eta)\| \\
& \leq a^{2}\|\Theta-\eta\|\|\mathcal{F}(\Theta)-\mathcal{F}(\eta)\| \\
& \leq \frac{a^{2}}{2}\|\Theta-\eta\|^{2}+\frac{a^{2}}{2}\|\mathcal{F}(\Theta)-\mathcal{F}(\eta)\|^{2}
\end{aligned}
$$

Using $k \geq 0$ results in

$$
\left(1+\frac{a^{2}}{2}\right)\|\mathcal{F}(\Theta)-\mathcal{F}(\eta)\|^{2} \leq \frac{a^{2}}{2}\|\Theta-\eta\|^{2} .
$$

Therefore,

$$
\|\mathcal{F}(\Theta)-\mathcal{F}(\eta)\|^{2} \leq \frac{a^{2}}{2+a^{2}}\|\Theta-\eta\|^{2}
$$

and $\mathcal{F}$ is a contraction on $S_{E}^{h}$. Thus, the sequence $\left\{\Theta_{l}^{n}\right\}$ defined by (2.3) converges to the unique solution of $(2.2 \mathrm{a})$ and $(2.2 \mathrm{~b})$ for any choice of $\Theta_{0}^{n}$.

Next, we derive an error bound for the approximation (2.2a) and (2.2b). We will use the projection $P_{E}^{h}: H_{E}^{1} \rightarrow S_{E}^{h}$ defined by

$$
\left(D P_{E}^{h} v, D \chi\right)=(D v, D \chi) \quad \forall \chi \in S_{E}^{h} .
$$

By [5] we have

$$
\begin{gathered}
P_{E}^{h} v\left(x_{i}\right)=v\left(x_{i}\right) i=0,1, \ldots, s \\
\left\|v-P_{E}^{h} v\right\|+h\left\|D v-D P_{E}^{h} v\right\| \leq C h^{2}\|v\|_{2} .
\end{gathered}
$$


Theorem 2.2. Let $\{\theta(t), \gamma(t)\}$ be the solution of $(1.7 \mathrm{a})-(1.7 \mathrm{e})$ and $\left\{\Theta^{n}, \Gamma^{n}\right\}$ be the solution of $(2.2 \mathrm{a})$ and $(2.2 \mathrm{~b})$. Then

$$
\Delta t \sum_{n=1}^{N}\left\|\Theta^{n}-\theta\left(t_{n}\right)\right\|^{2} \leq C(\theta, T)\left(h^{4}+(\Delta t)^{2}\right)
$$

and, as a consequence,

$$
\begin{aligned}
& \left\|\Theta^{n}-\theta\left(t_{n}\right)\right\| \leq C(\theta, T)\left(\frac{h^{2}}{(\Delta t)^{1 / 2}}+(\Delta t)^{1 / 2}\right), \\
& \left|\Gamma^{n}-\gamma\left(t_{n}\right)\right| \leq C(\theta, T)\left(\frac{h^{2}}{(\Delta t)^{1 / 2}}+(\Delta t)^{1 / 2}\right),
\end{aligned}
$$

with $C$ a constant independent of $h$ and $\Delta t$ and $t_{n}=n \Delta t$.

Proof. Observe that we need only derive the first estimate. Let $\theta^{n} \equiv \theta\left(t_{n}\right)$ and $\gamma^{n} \equiv \gamma\left(t_{n}\right)$. Integrating equation (2.1) from 0 to $t_{n}$ and summing (2.2a) from 1 to $n$, we obtain $\forall \chi \in S_{E}^{h}$

$$
\left(1+a^{2}\right)\left(\theta^{n}, \chi\right)-\left(1+a^{2}\right)\left(\theta_{0}, \chi\right)+\left(D \hat{\theta}^{n}, D \chi\right)+k \chi(1)\left(\int_{0}^{t_{n}} \theta(1, t) d t\right)
$$$$
\left(1+a^{2}\right)\left(\Theta^{n}, \chi\right)-\left(1+a^{2}\right)\left(\Theta^{0}, \chi\right)+\left(\Delta t \sum_{i=1}^{n} D \Theta^{i}, D \chi\right)+\Delta t k \chi(1) \sum_{i=1}^{n} \Theta^{i}(1)
$$

$$
=t_{n} \theta_{A} \chi(1)+a^{2}\left(\left[\Gamma^{n}\right]_{+}-\left[\Gamma^{0}\right]_{+}\right)(1, \chi),
$$

where $\hat{\theta}^{n}=\int_{0}^{t_{n}} \theta(s) d s$. Thus, using (2.5), we have $\forall \chi \in S_{E}^{h}$

$$
\begin{aligned}
& \left(1+a^{2}\right)\left(\Theta^{n}-\theta^{n}, \chi\right)-\left(D\left(P_{E}^{h} \hat{\theta}^{n}-\Delta t \sum_{i=1}^{n} \Theta^{i}\right), D \chi\right) \\
& +k \chi(1)\left(\Delta t \sum_{i=1}^{n} \Theta^{i}(1)-\hat{\theta}^{n}(1)\right)=a^{2}\left(\left[\Gamma^{n}\right]_{+}-\left[\gamma^{n}\right]_{+}, \chi\right) .
\end{aligned}
$$

Let us define

$$
\begin{aligned}
\varepsilon^{j} & =\Delta t \sum_{i=1}^{j} \Theta^{i}-P_{E}^{h} \hat{\theta}^{j}, \quad j=1, \ldots, n, \\
\varepsilon^{0} & =0, \\
\bar{\theta}^{j} & =\frac{1}{\Delta t} \int_{t_{j-1}}^{t_{j}} \theta(s) d s, \quad j=1, \ldots, n .
\end{aligned}
$$

It follows that

$$
\frac{\varepsilon^{j}-\varepsilon^{j-1}}{\Delta t}=\Theta^{j}-P_{E}^{h} \bar{\theta}^{j}
$$

and by $(2.9), \forall \chi \in S_{E}^{h}$

$$
\left(1+a^{2}\right)\left(\frac{\varepsilon^{n}-\varepsilon^{n-1}}{\Delta t}, \chi\right)+\left(D \varepsilon^{n}, D \chi\right)+k \chi(1)\left(\Delta t \sum_{i=1}^{n} \Theta^{i}(1)-\hat{\theta}^{n}(1)\right)=I_{1}+I_{2},
$$


where

$$
I_{1}=\left(1+a^{2}\right)\left(\theta^{n}-P_{E}^{h} \bar{\theta}^{n}, \chi\right)
$$

and

$$
I_{2}=a^{2}\left(\left[\Gamma^{n}\right]_{+}-\left[\gamma^{n}\right]_{+}, \chi\right) .
$$

Thus

$\left(1+a^{2}\right)\left(\frac{\varepsilon^{n}-\varepsilon^{n-1}}{\Delta t}, \chi\right)+\left(D \varepsilon^{n}, D \chi\right)+k \chi(1)\left(\varepsilon^{n}(1)+P_{E}^{h} \hat{\theta}^{n}(1)-\hat{\theta}^{n}(1)\right)=I_{1}+I_{2}$ and (2.6a) implies that

$$
\left(1+a^{2}\right)\left(\frac{\varepsilon^{n}-\varepsilon^{n-1}}{\Delta t}, \chi\right)+\left(D \varepsilon^{n}, D \chi\right)+k \chi(1) \varepsilon^{n}(1)=I_{1}+I_{2} .
$$

By applying the Cauchy-Schwarz inequality, we have

$$
\begin{aligned}
I_{1} & =\left(1+a^{2}\right)\left(\theta^{n}-\bar{\theta}^{n}+\bar{\theta}^{n}-P_{E}^{h} \bar{\theta}^{n}, \chi\right) \\
& =\left(1+a^{2}\right)\left(\frac{1}{\Delta t} \int_{t_{n-1}}^{t_{n}}\left(s-t_{n-1}\right) \theta_{t}(s) d s+\frac{1}{\Delta t} \int_{t_{n-1}}^{t_{n}}\left(\theta(s)-P_{E}^{h} \theta(s)\right) d s, \chi\right) \\
& \leq\left(1+a^{2}\right)\|\chi\| A_{n-1},
\end{aligned}
$$

where

$$
A_{n-1}=\frac{1}{\Delta t}\left(\left(\frac{(\Delta t)^{3}}{3} \int_{t_{n-1}}^{t_{n}}\left\|\theta_{t}(s)\right\|^{2} d s\right)^{\frac{1}{2}}+\left(\Delta t \int_{t_{n-1}}^{t_{n}}\left\|\theta(s)-P_{E}^{h} \theta(s)\right\|^{2} d s\right)^{\frac{1}{2}}\right)
$$

and

$$
\begin{aligned}
I_{2} & \leq a^{2}\|\chi\|\left\|\Theta^{n}-\theta^{n}\right\| \\
& \leq a^{2}\|\chi\|\left\|\Theta^{n}-P_{E}^{h} \bar{\theta}^{n}+P_{E}^{h} \bar{\theta}^{n}-\theta^{n}\right\| \\
& \leq A_{n-1} a^{2}\|\chi\|+a^{2}\|\chi\|\left\|\frac{\varepsilon^{n}-\varepsilon^{n-1}}{\Delta t}\right\| .
\end{aligned}
$$

Taking $\chi=\frac{\varepsilon^{n}-\varepsilon^{n-1}}{\Delta t}$ in $(2.10)$ results in

$$
\begin{gathered}
\frac{1+a^{2}}{(\Delta t)^{2}}\left\|\varepsilon^{n}-\varepsilon^{n-1}\right\|^{2}+\frac{1}{\Delta t}\left(D \varepsilon^{n}, D\left(\varepsilon^{n}-\varepsilon^{n-1}\right)\right)+\frac{k}{\Delta t}\left(\varepsilon^{n}(1)-\varepsilon^{n-1}(1)\right) \varepsilon^{n}(1) \\
\leq \frac{a^{2}}{(\Delta t)^{2}}\left\|\varepsilon^{n}-\varepsilon^{n-1}\right\|^{2}+\frac{C}{\Delta t}\left\|\varepsilon^{n}-\varepsilon^{n-1}\right\| A_{n-1} .
\end{gathered}
$$

Therefore,

$$
\begin{aligned}
\frac{1}{(\Delta t)^{2}}\left\|\varepsilon^{n}-\varepsilon^{n-1}\right\|^{2}+\frac{1}{2 \Delta t}\left(\left|\varepsilon^{n}-\varepsilon^{n-1}\right|_{1}^{2}+\left|\varepsilon^{n}\right|_{1}^{2}-\left|\varepsilon^{n-1}\right|_{1}^{2}\right) \\
\quad+\frac{k}{2 \Delta t}\left(\varepsilon^{n}(1)-\varepsilon^{n-1}(1)\right)^{2}+\frac{k}{2 \Delta t}\left(\left(\varepsilon^{n}(1)\right)^{2}-\left(\varepsilon^{n-1}(1)\right)^{2}\right) \\
\leq \frac{1}{2(\Delta t)^{2}}\left\|\varepsilon^{n}-\varepsilon^{n-1}\right\|^{2}+C A_{n-1}^{2}
\end{aligned}
$$


and

$$
\begin{gathered}
\frac{1}{(\Delta t)^{2}} \sum_{j=1}^{n}\left\|\varepsilon^{j}-\varepsilon^{j-1}\right\|^{2}+\frac{1}{\Delta t} \sum_{j=1}^{n}\left|\varepsilon^{j}-\varepsilon^{j-1}\right|_{1}^{2}+\frac{1}{\Delta t}\left|\varepsilon^{n}\right|_{1}^{2} \\
+\frac{k}{\Delta t} \sum_{j=1}^{n}\left(\varepsilon^{j}(1)-\varepsilon^{j-1}(1)\right)^{2}+\frac{k}{\Delta t}\left(\varepsilon^{n}(1)\right)^{2} \\
\leq \frac{1}{\Delta t}\left|\varepsilon^{0}\right|_{1}^{2}+\frac{k}{\Delta t}\left(\varepsilon^{0}(1)\right)^{2}+C \sum_{j=1}^{n} A_{j-1}^{2} .
\end{gathered}
$$

Since $k \geq 0, \varepsilon^{0}=0$ and $(2.6 \mathrm{~b})$ holds, the regularity result given by Theorem 1.1 and the definition of $A_{j-1}$ yields

$$
\Delta t \sum_{j=1}^{n}\left\|\frac{\varepsilon^{j}-\varepsilon^{j-1}}{\Delta t}\right\|^{2}+\left|\varepsilon^{n}\right|_{1}^{2}+k\left(\varepsilon^{n}(1)\right)^{2} \leq C(\theta, T)\left(h^{4}+(\Delta t)^{2}\right) .
$$

Noting that

$$
\Theta^{n}-\theta\left(t_{n}\right)=\Theta^{n}-P_{E}^{h} \bar{\theta}^{n}+P_{E}^{h} \bar{\theta}^{n}-\theta\left(t_{n}\right)
$$

the result follows.

\section{The Finite ELEMENT APPRoXimation of $u$}

A natural approximation to

$$
u\left(x, t_{n}\right)=-a x\left[\gamma\left(t_{n}\right)\right]_{+}+a \int_{0}^{x} \theta\left(\xi, t_{n}\right) d \xi+a \theta_{A}\left(x-\frac{x^{2}}{2}\right)
$$

is given by $U^{n} \in K^{h}$, where $K^{h}=\left\{v \in C^{0}(\bar{I}):\left.v\right|_{I_{j}} \in P_{1}, v(0)=0, v(1) \leq g\right\}$, defined by

$$
U^{n}\left(x_{j}\right)=-a x_{j}\left[\Gamma^{n}\right]_{+}+a \int_{0}^{x_{j}} \Theta^{n}(x) d x+a \theta_{A}\left(x_{j}-\frac{x_{j}^{2}}{2}\right), \quad j=1, \ldots, s,
$$

satisfying the following error bound.

Theorem 3.1. Suppose that $u\left(t_{n}\right)$ is given by (3.1). Then

$$
\left|u\left(x_{j}, t_{n}\right)-U^{n}\left(x_{j}\right)\right| \leq C(\theta, T)\left(\frac{h^{2}}{(\Delta t)^{1 / 2}}+(\Delta t)^{1 / 2}\right) .
$$

Proof. Observe that

$$
u\left(x_{j}, t_{n}\right)-U^{n}\left(x_{j}\right)=a x_{j}\left(\left[\Gamma^{n}\right]_{+}-\left[\gamma^{n}\right]_{+}\right)+a \int_{0}^{x_{j}}\left(\theta^{n}(x)-\Theta^{n}(x)\right) d x
$$

and so

$$
\begin{aligned}
\left|u\left(x_{j}, t_{n}\right)-U^{n}\left(x_{j}\right)\right| & \leq a\left|\left[\Gamma^{n}\right]_{+}-\left[\gamma^{n}\right]_{+}\right|+a\left\|\theta^{n}-\Theta^{n}\right\| \\
& \leq 2 a\left\|\theta^{n}-\Theta^{n}\right\| .
\end{aligned}
$$

The result is now a consequence of the error bound for the approximation of the temperature. 


\section{The STeady-STAte}

The stationary problem in (1.1a) $-(1.1 \mathrm{~h})$ is to find $\{\hat{\theta}(x), \hat{u}(x)\}$ such that

$$
\begin{array}{ll}
D^{2} \hat{\theta}=0, & x \in I, \\
D^{2} \hat{u}=a D \hat{\theta}, & x \in I, \\
\hat{\theta}(0)=\theta_{A}, & \\
-D \hat{\theta}(1)=k \hat{\theta}(1), & \\
\hat{u}(0)=0, & \\
\hat{u}(1) \leq g, \quad D \hat{u}(1) \leq a \hat{\theta}(1), & \\
(D \hat{u}(1)-a \hat{\theta}(1))(\hat{u}(1)-g)=0 . &
\end{array}
$$

We can see that $\{\hat{\theta}, \hat{u}\}$ given by

$$
\begin{gathered}
\hat{\theta}(x)=\theta_{A}+\left(\hat{\theta}(1)-\theta_{A}\right) x, \\
\hat{u}(x)=a \theta_{A} x+\frac{a}{2}\left(\hat{\theta}(1)-\theta_{A}\right) x^{2} \quad \text { if } \frac{a}{2}\left(\hat{\theta}(1)+\theta_{A}\right)<g, \\
\hat{u}(x)=\left(\hat{\sigma}+a \theta_{A}\right) x+\frac{a}{2}\left(\hat{\theta}(1)-\theta_{A}\right) x^{2} \quad \text { if } \frac{a}{2}\left(\hat{\theta}(1)+\theta_{A}\right)>g,
\end{gathered}
$$

where $\hat{\sigma}=D \hat{u}(1)-a \hat{\theta}(1)$, is the solution to this problem, and

$$
\begin{gathered}
\hat{\theta}(1)=\frac{\theta_{A}}{1+k}, \\
\hat{u}(1)=\min \left\{\frac{a}{2}\left(\hat{\theta}(1)+\theta_{A}\right), g\right\} .
\end{gathered}
$$

Hence, for fixed $a, k$ and $g$, there is contact or not depending on $\theta_{A}$. Taking $\theta_{A}>0$, it follows that $0<\hat{\theta}(1) \leq \theta_{A}$ and therefore, at the steady-state, contact will not be observed for $g>a \theta_{A}$. If $g<\frac{a}{2} \theta_{A}$ there will be contact with the obstacle.

When $k=0$ we have $\hat{\theta}(1)=\theta_{A}$ and $\hat{u}(1)=\min \left\{a \theta_{A}, g\right\}$. In the limit case, $k \rightarrow+\infty$, we find that

$$
\begin{aligned}
& \hat{\theta}(x) \rightarrow \theta_{A}(1-x), \\
& \hat{\theta}(1) \rightarrow 0, \\
& -D \hat{\theta}(1) \rightarrow \theta_{A}, \\
& \hat{u}(1) \rightarrow \min \left\{\frac{a}{2} \theta_{A}, g\right\}, \\
& \hat{\sigma} \rightarrow \min \left\{g-\frac{a}{2} \theta_{A}, 0\right\},
\end{aligned}
$$

with no temperature difference between the end $B$ and the obstacle.

\section{NUMERICAL EXPERIMENTS}

In our numerical simulations we took $\Delta t=h^{2}, a=0.017$ and $g=0.1$; the value for $a$ was taken from the work by Gilbert et al., in [9]. As an initial guess to $\Theta^{n}$ we choose $\Theta^{n-1}$ and the iterative process (2.3) was stopped when the difference between successive iterates was less than or equal to $1.0 \times 10^{-7}$. We let $h=\frac{1}{101}$, $p(x)=\theta_{A} \cos 2 \pi x$ and $\Theta^{0}$ was the interpolant of $\theta_{0}$. Numerical integration, namely the trapezoidal rule, was used to compute $M$ with the resulting matrix $M$ being diagonal with diagonal elements $M_{i i}=h, i \neq s, M_{s s}=\frac{h}{2}$. Note that the temperature $\tilde{\theta}$ is shown in the pictures. 

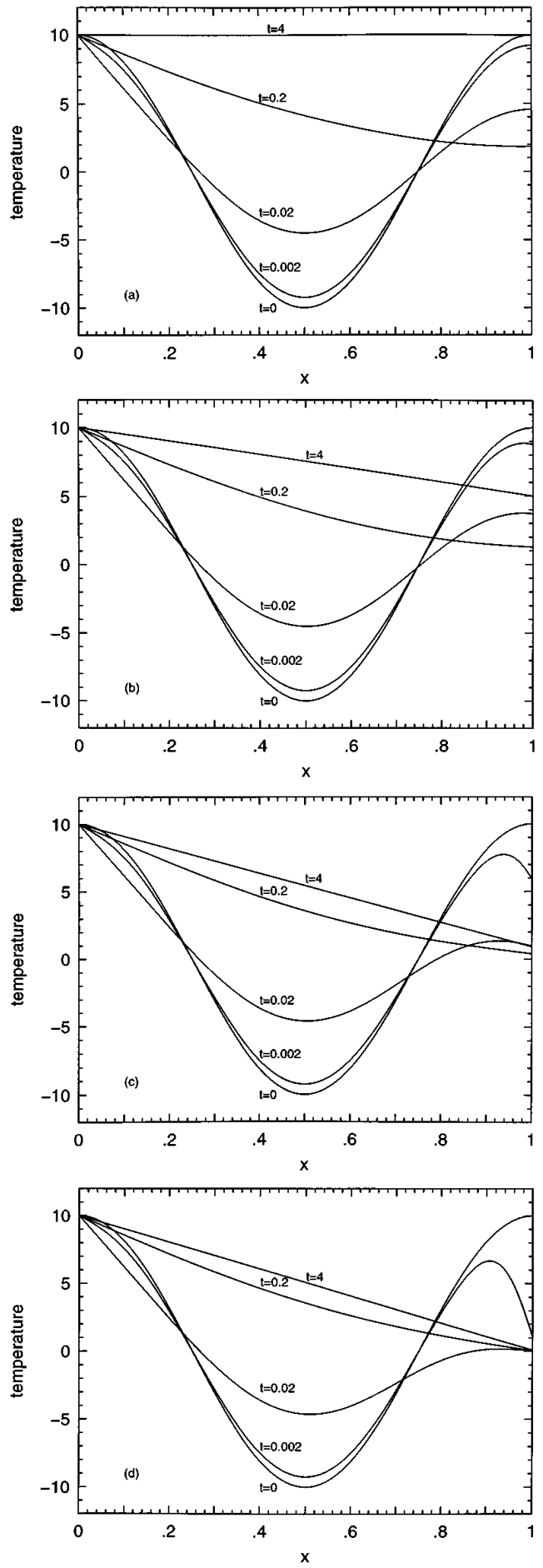

Figure 1. The evolution in time of the temperature from the initial condition when $\theta_{A}=10$ for (a) $k=0$, (b) $k=1$, (c) $k=10$ and (d) $k=100$. 

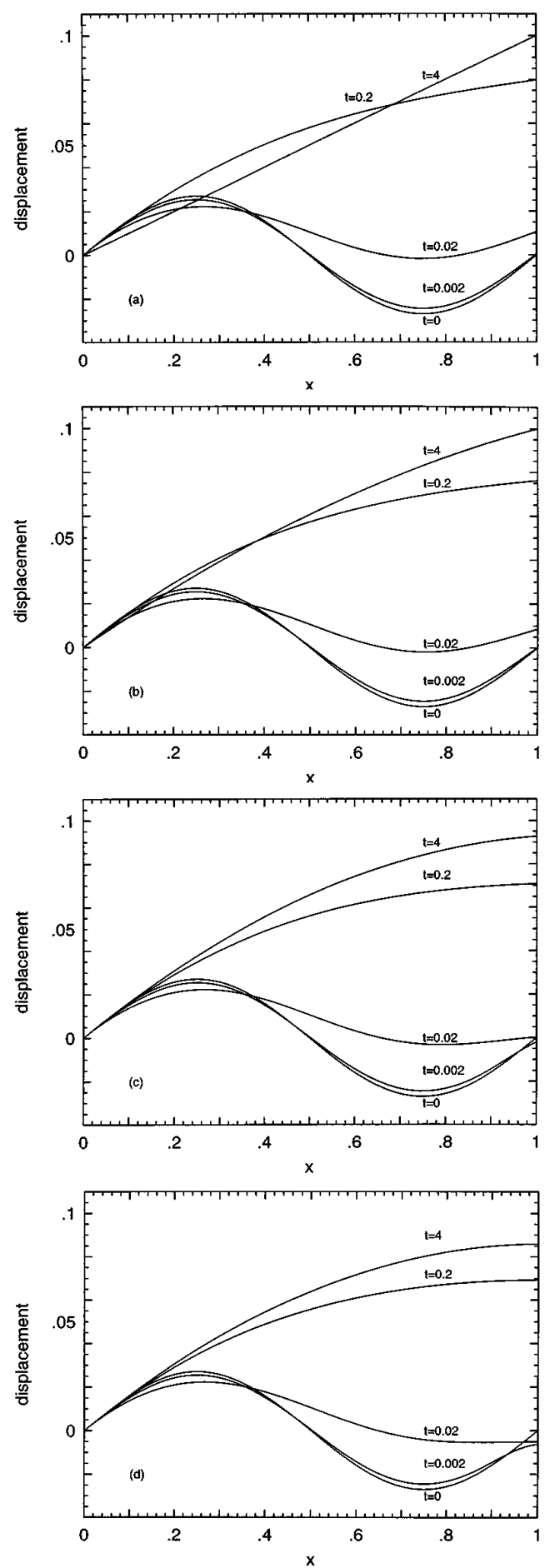

Figure 2. The evolution in time of the displacement when $\theta_{A}=$ 10 for (a) $k=0$, (b) $k=1$, (c) $k=10$ and (d) $k=100$. 
To investigate the convergence to the steady-state solution and the contact condition we performed four experiments. The numerical results are presented in Figures 1 and 2 where the temperature and the displacement are shown for $t=0,0.002$, $0.02,0.2$ and 4 . The graphs did not change after the final state shown and the computations were stopped.

We fixed $\theta_{A}=10$ and took increasing values of the heat transfer coefficient $k$, $k=0,1,10$ and 100. Contact is observed when $k=0$ and $k=1$ and for $k=100$, $\tilde{\theta}(1)$ is very small.

In all simulations, the numerical results are in agreement with the theoretical results given above.

\section{ACKNOWLEDGMENT}

The author wishes to thank the referee for his valuable suggestions.

\section{REFERENCES}

[1] K. T. Andrews, P. Shi, M. Shillor and S. Wright, Thermoelastic contact with Barber's heat exchange condition, Appl. Math. Optim., 28, 1993, 11-48. MR 94e:73051

[2] B. A. Boley and J. H. Weiner, Theory of thermal stresses, John Wiley, 1960. MR 22:3265

[3] D. E. Carlson, Linear thermoelasticity, in Handbuch der physik, (ed. C. Truesdell), vol. VIa/2, 297-345, 1972.

[4] M. I. M. Copetti and C. M. Elliott, A one-dimensional quasi-static contact problem in linear thermoelasticity, Euro. Jnl. Appl. Math., 4, 1993, 151-174. MR 94i:73079

[5] M. Crouzeix and J. Rappaz, On numerical approximation in bifurcation theory, Masson, 1990. MR 92d:65003

[6] W. A. Day, Heat conduction within linear thermoelasticity, Springer, New York, 1985. MR 87c:73001

[7] G. Duvaut, Free boundary problem connected with thermoelasticity and unilateral contact, in Free boundary problems vol. II, pp. 217-236, Rome, 1980. MR 83g:73013

[8] C. M. Elliott and T. Qi, A dynamic contact problem in thermoelasticity, Nonlinear Anal., 23, 1994, 883-898. MR 95i:73013

[9] R. P. Gilbert, P. Shi and M. Shillor, A quasistatic contact problem in linear thermoelasticity, Rediconti di Matematica, 10, 1990, 785-808. MR 92m:73109

[10] P. Shi and M. Shillor, Uniqueness and stability of the solution to a thermoelastic contact problem, Euro. J. Appl. Math., 1, 1990, 371-387. MR 92f: 73010

[11] P. Shi, M. Shillor and X. Zou: Numerical solutions to one dimensional problems of thermoelastic contact, Comput. Math. Appl., 22, 1991, 65-78. MR 92k:73064

Departamento de Matemática, Universidade Federal de Santa Maria, 97119-900 Santa MARIA, RS, BRASIL

E-mail address: mimc@lana.ccne.ufsm.br 